\title{
Verification of Hybrid Systems
}

\author{
Frits Vaandrager \\ Computing Science Institute \\ University of Nijmegen \\ P.O. Box 9010, 6500 GL Nijmegen \\ The Netherlands \\ Frits.Vaandrager@cs.kun.nl
}

\begin{abstract}
The next stage of the computer revolution consists in the proliferation of sophisticated and cheap digital controllers into almost every aspect of man-made systems. Informatics is expected to shift its focus of attention from computers performing internal computations, or communicating with human users and with other computers, toward computers interacting in real-time with physical processes. In such settings, the proper functioning of the whole system depends critically on the interaction between the discrete dynamics of the digital controller and the continuous dynamics of the environment in which it is embedded. Models of hybrid systems suggest a framework for modelling, simulation, verification, synthesis and implementation of such systems.

The main activity of the Esprit LTR project VHS - Verification of Hybrid Systems - consists of analysing academic and industrial case studies, taken from the process control industry, in order to define formal models of plants. These models are then used to verify properties concerning their behaviour. The project uses, among others, the models of timed and hybrid automata to express hybrid phenomena. Several tools for analysing systems expressed in this formalism have been built, and are used within the project for automatic verification.

The VHS project started in 1998. The consortium is composed of five CS partners (Verimag, Weizmann, Nijmegen, Brics and Kiel), two chemical engineering and process control partners (Dortmund, LAG), two partners from control theory (CWI and Ghent) and three industrial partners (Sidmar, Nylstar, Krupp).

In this talk, I will report on some initial results obtained by the project, and discuss the challenges ahead of us.
\end{abstract}

